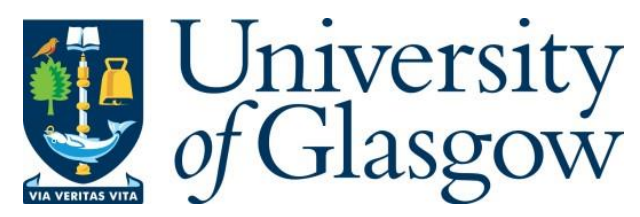

Cerezo Sanchez, M., Zuo, S., Moldovan, A., Cochran, S., Nazarpour, K. and Heidari, H. (2021) Flexible Piezoelectric Sensors for Miniaturized Sonomyography. In: 2021 43rd Annual International Conference of the IEEE Engineering in Medicine and Biology Society (EMBC), 01-05 Nov 2021, pp. 7373-7376. ISBN 9781728111797.

There may be differences between this version and the published version. You are advised to consult the publisher's version if you wish to cite from it.

http://eprints.gla.ac.uk/247167/

Deposited on: 22 July 2021

Enlighten - Research publications by members of the University of Glasgow http://eprints.gla.ac.uk 


\title{
Flexible Piezoelectric Sensors for Miniaturized Sonomyography
}

\author{
Maria Cerezo Sanchez, Student Member, IEEE, Siming Zuo, Student Member, IEEE, \\ Alexandru Moldovan, Member, IEEE, Sandy Cochran, Senior Member, IEEE, Kianoush Nazarpour, \\ Senior Member, IEEE, and Hadi Heidari, Senior Member, IEEE
}

\begin{abstract}
Sonomyography refers to the measurement of muscle activity with an ultrasonic transducer. It is a candidate modality for applications in diagnosis of muscle conditions, rehabilitation engineering and prosthesis control as an alternative to electromyography. We propose a mechanicallyflexible piezoelectric sonomyography transducer. Simulating different components of the transducer, using COMSOL Multiphysics $^{\circledR}$ software, we analyze various electromechanical parameters, such as von Mises stress and charge accumulation. Our findings on modelling of a single-element device, comprised of a PZT-5H layer of thickness $66 \mu \mathrm{m}$, with a polymer substrate $(E=2.5 \mathrm{GPa})$, demonstrate optimal flexibility and charge accumulation for sonomyography. The addition of Polyimide and PMMA as an acoustic matching layer and an acoustic lens, respectively, allowed for adequate energy transfer to the medium, whilst still maintaining good mechanical properties. In addition, preliminary ultrasound transmission simulations (200 $\mathrm{kHz}-30 \mathrm{MHz}$ ) showed the importance of the aspect ratio of the device and how there is a need for further studies on it. The development of such a technology could be of great use within the healthcare sector, not only due to its ability to provide highly accurate and varied real-time muscle data, but also because of the range of applications that could benefit from its use.
\end{abstract}

\section{INTRODUCTION}

The study of muscle activity dates back to the early decades of the $20^{\text {th }}$ century, when the principles of electromyography (EMG) were established. Ever since, this type of functional and anatomical study has been developed and improved to diagnose neuromuscular conditions. Recently, along with these developments in EMG methods, novel techniques that allow for the concurrent study of muscle structure and function with higher spatial resolution than that with the EMG method have emerged, e.g. magnetomyography $[1,2]$ and sonomyography (SMG) [3].

SMG refers to the use of ultrasonic waves to investigate muscle structure and function. This technique was first described in the early 2000s by Zheng et al. [3] where they aimed to use SMG to replace EMG-controlled prostheses. This research also pointed out some of the main disadvantages of using EMG, such as its inability to differentiate between the muscle movement of the main muscle and that of neighbouring muscles as well as its inefficiency scanning deeper muscles. This is where SMG could prove to be a better alternative. Other studies have also highlighted its potential as an alternative to the traditionally used electromyography since it

This work was supported by grant EP/R511705/1 from EPSRC, UK. The work of $\mathrm{KN}$ is supported by EPSRC grant EP/R004242/1.

CS. Maria, S. Zuo, M. Alexandru, C. Sandy and H. Heidari are with the James Watt School of Engineering, University of Glasgow, G12 8QQ, UK. (e-mail: 2246671C@student.gla.ac.uk and Hadi.Heidari@glasgow.ac.uk).

K. Nazarpour is with the School of Informatics, University of Edinburgh, EH8 9AB, UK. (e-mail: kianoush.nazarpour@ed.ac.uk).

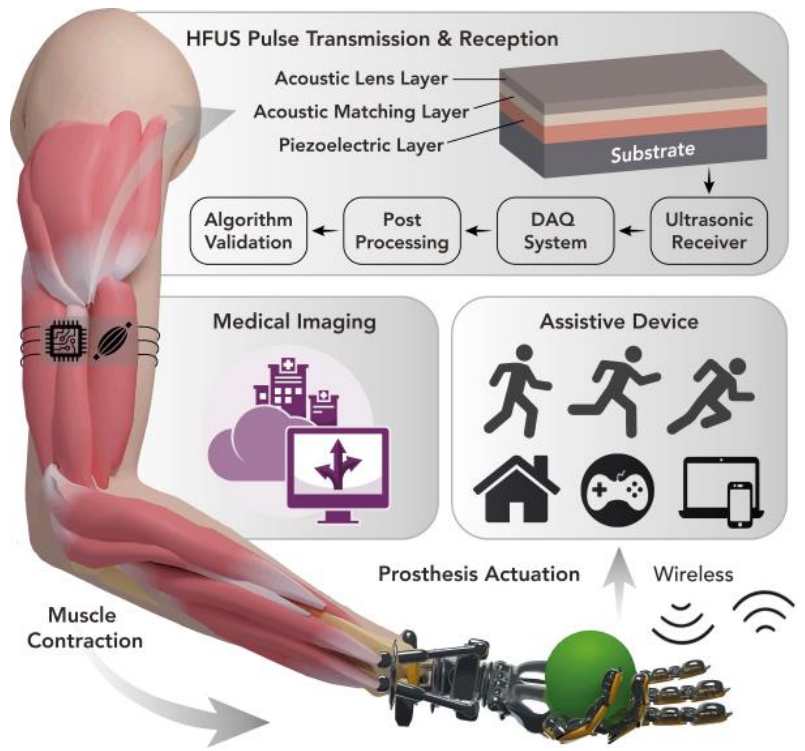

Fig. 1. An overall architecture of the flexible, HFUS sonomyography device. The two main applications: medical imaging, ranging from the diagnosis and analysis of myopathies to spinal cord lesions, and assistive devices, aiding in the control of prosthetic limbs and muscle-computer interfaces.

can produce very robust signals and can be highly specific [4]. Moreover, ultrasound technologies are currently one of the most researched areas within the biomedical imaging sector. Therefore, this type of technology can potentially allow a major improvement in the quality of the produced images while reducing the costs and complexity of the required devices.

Furthermore, studies have shown the potential of flexible piezoelectric films within the nanogenerators and energy harvesting sector, but there is a general lack of research within the biomedical engineering sector. Few studies have investigated the use of high frequency ultrasound (HFUS) to enhance the spatial resolution of sonomyography [5]. This poses an opportunity to investigate the potential of combining flexible transducers and HFUS.

We aim to illustrate the potential of sonomyography as an imaging and assistive technique for assessing muscle activity. This method could lead to a more efficient and accurate diagnosis of myopathies and damage to the peripheral nervous system and optimizing assistive and rehabilitation engineering techniques. We, therefore, designed a flexible and miniaturized HFUS transducer and simulated each stage of the design process with COMSOL Multiphysics $®$ to validate the model that can be used in future SMG applications. Fig. 1 illustrates the proposed design of the overall device, including the flexible transducer and a simple block diagram detailing the electronics and post-processing for the system. 


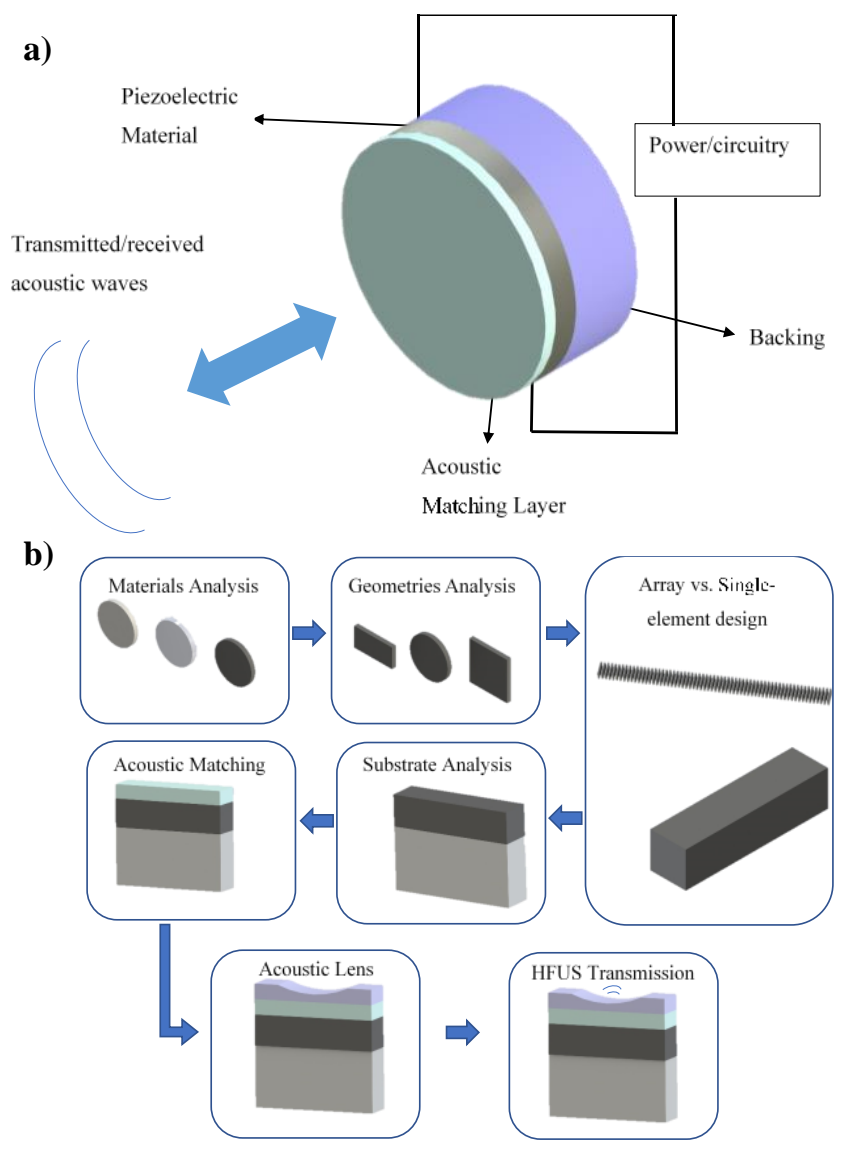

Fig. 2. (a) Single-element transducer design; (b) Detailed methodology for the device's design from the material to HFUS transmission analysis.

\section{Piezoelectric Sensor Design}

\section{A. Structure and Principle}

Ultrasound can be defined as "acoustic waves (sound or pressure waves) propagating within a matter medium at frequencies exceeding the auditory band" [6]. The piezoelectric effect [7], details how certain materials demonstrate an accumulation of electrical charge when a mechanical force or stress is applied to them. The inverse piezoelectric effect indicates that a piezoelectric material deforms when excited with an external electric field. This effect allows transmission and reception of ultrasound waves with piezoelectric transducers.

A simple schematic of a single-element ultrasound transducer is shown in Fig. 2 (a). A key consideration is the choice of the piezoelectric material. Crystalline and ceramics are common piezoelectric materials but the most commonly used piezoceramic is lead zirconate titanate (PZT) [5] due to its high electromechanical coupling coefficient and its very adequate dielectric and elastic properties for biomedical imaging. Other materials of interest inlude polyvinylidene fluoride (PVDF), shown to have great flexibility and biocompatibility [8], and Aluminium Nitride (AIN) which offers higher sensitivity than PZT [9]. These materials were analysed in different studies (Table 1) to evaluate the optimum configuration for SMG applications. In addition, the dimensions and geometries of the piezoelectric element were investigated, with circular, square and rectangular geometries analyzed, as commonly perfomed in the literature $[10,11]$.
Table 1. State-of-the-art studies on flexible piezoelectric sensors

\begin{tabular}{ccccc}
\hline \hline $\begin{array}{c}\text { Frequency } \\
\text { range }\end{array}$ & [10] & [8] & [9] & This study \\
\hline $\begin{array}{c}\text { Piezoelectric } \\
\text { material }\end{array}$ & PZT & PVDF & AlN & PZT \\
\hline Thickness & $0.4-1.5 \mathrm{~mm}$ & $\begin{array}{c}52 \mu \mathrm{m}(2 \\
\text { layers })\end{array}$ & $1 \mu \mathrm{m}$ & $66-100 \mu \mathrm{m}$ \\
\hline Dimensions & $\begin{array}{c}\text { Relies on } \\
\text { FES, low } \\
\text { are too } \\
\text { large for } \\
\text { HFUS }\end{array}$ & $\begin{array}{c}\text { Sensitivity } \\
\text { at high } \\
\text { frequency }\end{array}$ & $\begin{array}{c}\text { pir, low } \\
\text { piezoelectric } \\
\text { coefficients }\end{array}$ & $\begin{array}{c}\text { Aspect } \\
\text { ratio and } \\
\text { foreign } \\
\text { body } \\
\text { response }\end{array}$ \\
\hline \hline
\end{tabular}

We evaluated the effect of the backing layer or substrate, the acoustic matching layer and the acoustic lens, as well as an array configuration versus a single-element geometry, as shown in Fig. 2 (b).

The backing layer of an ultrasonic transducer allows for reverberation to be damped and is bonded to the piezoelectric material, as shown in Fig. 2. A mixture of Tungsten powder and a soft-setting epoxy tends to be the common choice within the sector due to its high acoustic attenuation [12]. However, this can challenge the flexibility of the sensor/transducer. There are three possible alternative substrates that serve as a backing for the device: silicon, polydimethylsiloxane (PDMS) and polyimide (PI) $[12,13]$.

Another component of great interest is the acoustic matching layer, which serves as an aid for energy transfer between the piezoelectric material and the surrounding medium, and vice versa. This allows for an improvement in the efficiency of the transducer. A literature review revealed that some studies have shown that certain polymer resins, such as PI, demonstrate satisfactory results when used in lower frequency ultrasonic devices and has the advantage of being quite flexible [14].

The final component of interest to the project is the acoustic lens. Acoustic lenses work in a similar way to optical ones by focusing the ultrasonic waves in order to create the desired beam shape. In terms of the investigated materials, according to the literature, polymethyl methacrylate (PMMA) and PI plano-concave lenses have shown adequate results in high-frequency ultrasound imaging and have the possibility of being micromachined, aiding in the fabrication of the micronscale components [15].

\section{B. Finite-Element Simulation}

In order to design such a transducer, a similar methodology to that provided in recent studies was followed [10], with an additional final stage to analyse the performance of the designed sensor as an ultrasonic transmitter and not just a receiver, as shown in Fig. 3. This was done by using the COMSOL Multiphysics ${ }^{\circledR}$ simulation package. The design process was divided into the stages shown in Fig. 2 (b), with the used parameters shown in Table 2.

The mechanical and electrical parameters of interest were the stress, the strain, the displacement, the electric potential and the charge produced when pressure was applied. In this case, all components were subjected to a pressure of $10 \mu \mathrm{Pa}$ and $10 \mathrm{~Pa}$, and compared to one another. In addition, the electrical impedance spectrum of the different transducer 
Table 2. Used parameters for simulations.

\begin{tabular}{ccc}
\hline \hline Frequency & $20 \mathrm{MHz}$ & $30 \mathrm{MHz}$ \\
\hline Piezoelectric material & $100 \mu \mathrm{m}$ & $66 \mu \mathrm{m}$ \\
\hline Substrate & $300 \mu \mathrm{m}$ & $198 \mu \mathrm{m}$ \\
\hline Acoustic matching layer & $12.5 \mu \mathrm{m}$ & $18.7 \mu \mathrm{m}$ \\
\hline Acoustic lens & $12.5 \mu \mathrm{m}$ & $18.7 \mu \mathrm{m}$ \\
\hline \hline
\end{tabular}

geometries and the safety factor of the substrates were analysed with Eq. (1) and (2), respectively:

$$
\begin{array}{ll}
\text { (1) } X_{C}=\frac{\delta}{2 \pi f \varepsilon A} & \text { (2) Safety factor }=\frac{\text { yield stress }}{\text { von mises stress }}
\end{array}
$$

where $X_{c}$ is the electrical impedance, $\delta$ is the thickness of the piezoelectric material, $f$ is the operating frequency, $\varepsilon$ is the relative permitivitty and $A$ is the surface area of the piezoelectric element.

\section{RESULTS}

Simulation results showed that even if PVDF has a higher flexibility than PZT-5H (Fig. 3 (a)) and a lower acoustic impedance, its sensitivity is reduced. This is mainly due to the fact that PVDF has a much lower k33 coefficient than PZT$5 \mathrm{H}$, as well as high dielectric loss. In terms of electrical parameters, charge amplifiers are a very common choice for the circuitry design of an ultrasonic transducer [16], meaning that obtaining the greatest charge accumulation was crucial and hence, illustrates the preference of PZT-5H over the other two materials (Fig. 4 (b)). This result was also obtained for the chosen rectangular geometry, as well as an adequate response regarding electrical impedance (Fig. 4(c) and (d)).

The final piezoelectric film was analysed further to evaluate the differences between a single-element and an array design. For the single-element design, the simulation was carried out on the same rectangular PZT-5H element as in the previous section, but this time with a thickness of $66 \mu \mathrm{m}$. This
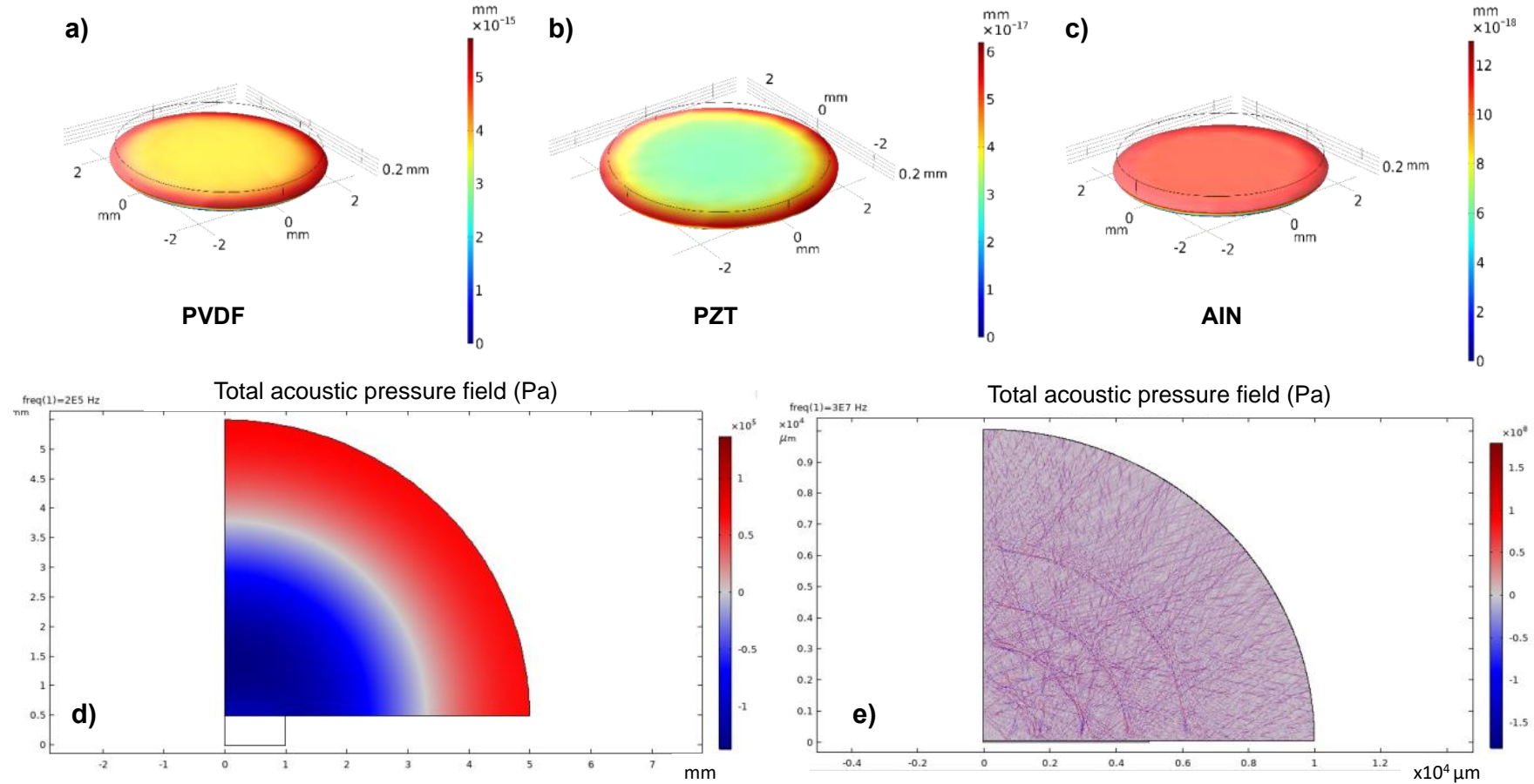

Fig. 3. Analysis of materials with (a) PVDF, (b) PZT and (c) AlN; (b) Acoustic pressure parameters analysed for a two-dimensional PZT rectangular film of dimensions $1 \times 0.5 \mathrm{~mm}$ and $5 \mathrm{~mm} \times 66 \mu \mathrm{m}$ at the frequency (c) $200 \mathrm{kHz}$ and (d) $30 \mathrm{MHz}$, respectively. thickness, corresponding to $30 \mathrm{MHz}$, was chosen for the rest of the simulations within the study because a higher frequency allows for better resolution. The decision on whether to use a single-element or an array design depends solely on the application of the sonomyography device. If the device were to be used for prosthesis control, then single-element transducers would be the ideal choice due to their simplicity, cost-effectiveness and accurate output information [5].

Regarding substrate analysis, a compromise between the safety factor and charge accumulation results was required (Fig.4 (e) and (f)), hence PI was the material of choice. For the acoustic matching layer, the thickness was the first parameter that needed to be calculated. In terms of the acoustic matching layer, since it has been demonstrated in the literature that an ideal acoustic layer thickness is $1 / 4$ the wavelength of interest [14]. Therefore, the thickness of the acoustic matching layer was calculated to be between $18.75 \mu \mathrm{m}$ and $12.5 \mu \mathrm{m}$ for a frequency range of $20-30 \mathrm{MHz}$, respectively, with an ideal acoustic impedance of $6.7 \mathrm{MRayl}$. In order to obtain such an acoustic impedance, it is common to chemically characterise the material of the matching layer by using powders containing different concentrations of materials such as Tungsten. This could be performed on a material like PI, which allows for flexibility and has been shown to perform well in highfrequency settings [14]. As for the acoustic lens, it was seen that a PMMA plano-concave lens with a radius of curvature of $7.4 \mu \mathrm{m}$, could allow for a simpler fabrication process and an effective transfer of energy to the medium.

In terms of the ultrasound transmission simulation results piezoelectric film prevented acoustic waves to be transmitted properly and should be revisited in future work. (Fig. 3 (d) and (e)), we showed that the high aspect ratio of the 

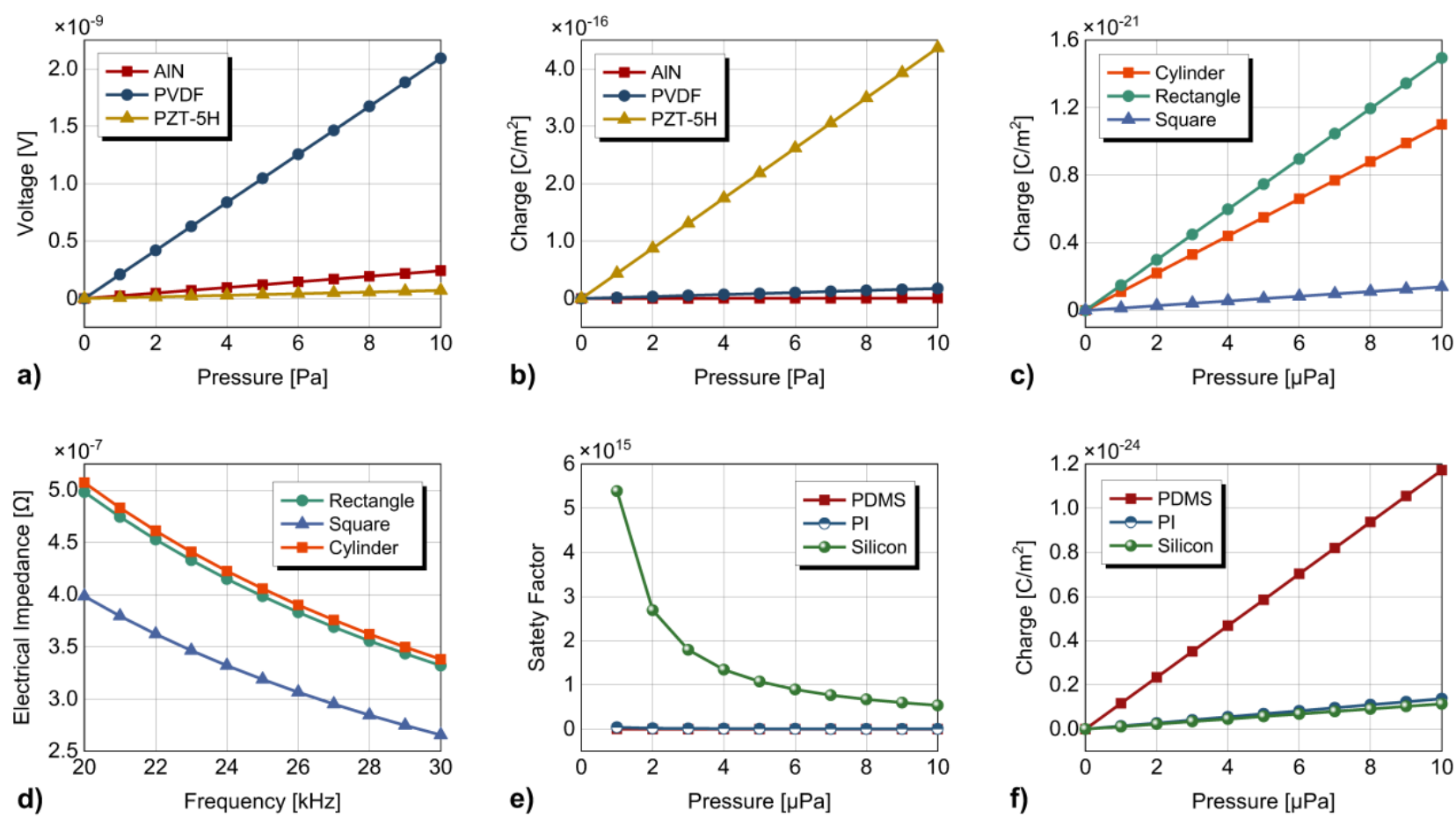

Fig.4. Comparison of electric potential and charge accumulation changes with increasing pressure for the three different materials: (a) Electric Potential (V); (b) Surface charge accumulation $\left(\mathrm{nC}^{2} \mathrm{~m}^{2}\right)$; Further analysis on MATLAB of the electrical impedance and the charge accumulation for the different geometries: (c) Surface charge accumulation $\left(\mathrm{nC}^{2} \mathrm{~m}^{2}\right)$ and (d) Electrical impedance-frequency response $(\Omega)$; (e) Safety factor for the three different substrates with changing pressure; (f) Surface charge accumulation for the three different substrates with changing pressure.

\section{CONCLUSIONS}

A new method for the study of muscle structure and function has been proposed through the use of a highfrequency flexible ultrasonic transducer. With COMSOL Multiphysics ${ }^{\circledR}$ simulations, it was demonstrated that a PZT5H transducer, with PI for the backing and acoustic matching layer and PMMA as a plano-concave acoustic lens, could allow for high resolution SMG. The application range of this sensor model can range from medical imaging to prosthetics and rehabilitation engineering. Further analysis on the aspect ratio, real-time performance, and micromachining fabrication techniques is required to determine whether SMG can replace EMG as a diagnostic and rehabilitation tool.

\section{REFERENCES}

[1] S. Zuo, et al., "Ultrasensitive Magnetoelectric Sensing System for picoTesla MagnetoMyoGraphy," IEEE Transactions on Biomedical Circuits and Systems, vol. 14, no. 5, pp. 971-984, 2020.

[2] S. Zuo, et al., "Miniaturized Magnetic Sensors for Implantable Magnetomyography", Advanced Materials Technologies, 2000185, 2020.

[3] Y. Zheng, M. Chan, J. Shi, X. Chen and Q. Huang, "Sonomyography: monitoring morphological changes of forearm muscles in actions with the feasibility for the control of powered prosthesis," Medical Engineering and Physics, vol. 28, no. 5, pp. 405-415, 2006.

[4] N. Akhlaghi, A. Dhawan, A. A. Khan, B. Mukherjee, G. Diao, C. Truong and S. Sikdar, "Sparsity analysis of a sonomyographic muscle-computer interface," IEEE Transactions on Biomedical Engineering, vol. 67, no. 3, pp. 688-696, 2020.

[5] J. A. Ketterling and R. H. Silverman, "Clinical and preclinical applications of high-frequency ultrasound," Acoustics Today, vol. 13, no. 1, pp. 44-51, 2017.

[6] H. Azhari, "Introduction: Prelude and Basic Definitions," in Basics of Biomedical Ultrasound for Engineers, New Jersey, Wiley-IEEE Press, 2010, pp. 1-7.
[7] J. Curie and P. Curie, "Development, via compression, of electric polarization in hemihedral crystals with inclined faces" Bulletin de la Société Minérologique de France, vol. 3, no. 4, pp. 90-93, 1880.

[8] I. Al-Mohimeed and Y. Ono, "Ultrasound measurement of skeletal muscle contractile parameters using flexible and wearable single-element ultrasonic sensor," Sensors, vol. 20, no. 13, p. 3616, 2020.

[9] E. Shin, et al., "Development of a high-density piezoelectric micromachined ultrasonic transducer array based on patterned aluminum nitride thin film," Micromachines, vol. 11, no. 6, p. 623, 2020.

[10] V. Pashaei, et al., "Flexible body-conformal ultrasound patches for image-guided neuromodulation," IEEE Trans Biomedical Circuits and Systems, vol. 14, no. 2, pp. 305-318, 2020.

[11] J. Jung, et al., "Review of piezoelectric micromachined ultrasonic transducers and their applications," J. Micromechanics Microengineering, vol. 27, no. 11, pp. 1-24, 2017.

[12] I. Ladabaum, et al., "Silicon substrate ringing in microfabricated ultrasonic transducers," in IEEE Ultrasonics Symposium. Proceedings. An International Symposium, San Juan, Puerto Rico, 2000.

[13] G. Galeote-Checa, et al., "Flexible wirelessly powered implantable device," in IEEE Int Conference Electronics, Circuits and Systems (ICECS), Genoa, Italy, 2019.

[14] J.-H. Park, et al., "Acoustic matching layer films using B-stage thermosetting polymer resins for Ultrasound Transducer Applications," IEEE Transactions Ultrasonics, Ferroelectrics, Frequency Control, vol. 67, no. 10, pp. 2148-2154, 2020.

[15] J. Li, et al., "Design and fabrication of a miniaturized, integrated, highfrequency acoustical lens-transducer system," J. Micromechanics Microengineering, vol. 12, 219-228, 2002.

[16] Y. Zhou, S. Lau, D. Wu and K. Shung, "Piezoelectric films for high frequency ultrasonic transducers in biomedical applications," Progress in Materials Science, vol. 56, pp. 139-174, 2011. 